\title{
Analysis of Static and Dynamic Properties of Micromirror with the Application of Response Surface Method
}

\author{
Adam Martowicz, Andrzej Klepka, Tadeusz Uhl \\ AGH University of Science and Technology, \\ Faculty of Mechanical Engineering and Robotics, \\ Department of Robotics and Mechatronics, \\ al. A. Mickiewicza 30, 30-059 Krakow, Poland \\ adam.martowicz@agh.edu.pl, klepka@agh.edu.pl, tuhl@agh.edu.pl
}

\begin{abstract}
The paper presents the results of an application of response surface method to aid the analysis of variation of static and dynamic properties of micromirror. The multiphysics approach was taken into account to elaborate finite element model of electrostatically actuated microdevice and coupled analyses were carried out to yield the results. Used procedure of metamodel fitting is described and its quality is discussed. Elaborated approximations were used to perform the sensitivity analysis as well as to study the propagation of variation introduced by uncertain and control parameters. The input parameters deal with geometry, material properties and control voltage. As studied output characteristics there were chosen the resultant static vertical displacement of reflecting surfaces and the resonance frequency related to the first normal mode of vibration.
\end{abstract}

Keywords: MEMS; micromirror; design process; FE model; multiphysics; response surface method; genetic algorithms; sensitivity analysis; uncertainty propagation.

\section{INTRODUCTION}

Nowadays there is observed rapid growth of application range of microelectromechanical systems (MEMS) resulting from their obvious advantages [1,2,3]. Manufacturing processes used to produce MEMS devices allow for the assembling mechanical parts with control electronic circuits together integrated in one package [4,5]. Manufactured microdevices are mainly constructed as silicon components acting both as micron-size movable mechanical parts, e.g. beams, membranes, rotating joints, racks, gears, etc., and embedded electronic circuits designed to control the motion of mechanical parts, amplify electrical signals, acquire measurement data and finally establish the interface for external communication with a user. The most known applications of MEMS technology are: accelerometers, gyroscopes, resonators, microphones, pumps, optic signal switchers, signal filters, microreactors, pressure, temperature and humidity sensors $[6,7,8]$. MEMS devices are applied in biology, chemistry, measurement techniques of non-electrical quantities, optics, automotive industry and aviation.

Microdevices are mostly manufactured with lithography-based techniques which are well-known and commonly applied for electronic circuits. In general MEMS components can be produced with bulk and surface micromachining $[9,10]$. Bulk micromachining deals 
with microstructuring carried inside a substrate, mostly made of mono-crystalline silicon wafer, by etching e.g. with potassium hydroxide. Surface micromachining in turn stands for the subsequent deposition and selective etching done with thin layers of different materials. Additionally, semi-final products made with both bulk and surface micromachining can also be bonded to establish final device [11]. The combination of above mentioned manufacturing techniques enables for the creation of suspended structures also of complicated shapes.

Widely used different MEMS devices also require many tests to be performed to elaborate designs with desired properties $[5,12,13]$. As it is of common engineering practice, the procedures of virtual prototyping, including finite element (FE) analyses, are carried out to investigate static and dynamic properties of microdevices. Numerical simulations are applied to perform sensitivity analyses, analyses of uncertainty propagation, design optimization as well as a study of reliability and performance of modelled MEMS structure $[7,14,15]$. Computer simulations including also FE analyses, amongst obvious advantages, feature some inconveniences. In case of MEMS devices it is common the existence of at least two different physical phenomena that have to be modelled, e.g. electrostatics or air damping phenomenon apart from classical formulations used to model structural dynamics. The approach which introduces several physical domains leads to quite complicated FE models prepared for coupled analyses. Multiphysics calculations can even be additionally complicated by nonlinearities. They extend the computational time considerably by the necessity of introduction of iterative procedures. The problem arises especially in a case when many realizations of designed device have to be checked to conclude about variation of studied model properties. There are however known and effectively applied approaches to obtain time savings:

- $\quad$ application of response surface method (RSM) to approximate investigated parameters and skip FE analyses $[8,16,17]$,

- modifications of used simulation methods to reduce required number of performed simulations to conclude about sensitivities or uncertainties, e.g. Monte Carlo simulation with improvement techniques of sample generation $[18,19,20]$,

- $\quad$ reduction or decomposition of FE model, which means either decrease of number of nodes, number of degrees of freedom (DOF) resulting in reduced sizes of global mass, damping and stiffness matrices or decomposition of system of matrix equation to be solved into a number of less complicated systems which can be separately solved faster and then used to combine final results [21],

- $\quad$ reduction of problem dimension which means either elimination of depended input parameters or reduction of number of input parameters considered in a study; in later case a sensitivity analysis is performed to keep only influential parameters [22],

- $\quad$ parallel computing performed with a cluster of computers [23],

- $\quad$ applications of hybrid methods to divide optimization task into two steps: first one with non-gradient method responsible for crude search of a region where global optimum is located and second one when gradient-based methods enable for fast and exact localization of optimal solution [24].

In the paper the results of static and dynamic simulations carried out for the FE model of electrostatically actuated micromirror are presented. The work focuses on the application of RSM used to speed-up calculations. Metamodelling technique is used to study the variation of static displacement and chosen natural frequency of vibration. Elaborated FE model considers multiphysics approach to introduce the presence of electrostatic field. 
The paper consists of seven sections. In section 2 the FE model is described. The input and output parameters for all performed analyses are described in section 3. Section 4 deals with the metamodelling technique including applied fitting strategy. Sections 5 and 6 present and discuss the results of sensitivity and uncertainty propagation analyses respectively. Final section summarizes the paper and presents concluding remarks.

\section{FE MODEL OF MICROMIRROR}

A micromirror was chosen for the study as it is presently one of the most commonly applied MEMS devices [25,26,27]. Micromirrors are used to direct a path of light ray and can act as switches to commute optic signals. They stand for an alternative to complicated electronic circuits with switching gates. The model of a simple micromirror was elaborated in the CoventorWare software [28]. The geometric model, main dimensions and masks considered for lithography-based manufacturing process are shown in Figure 1.
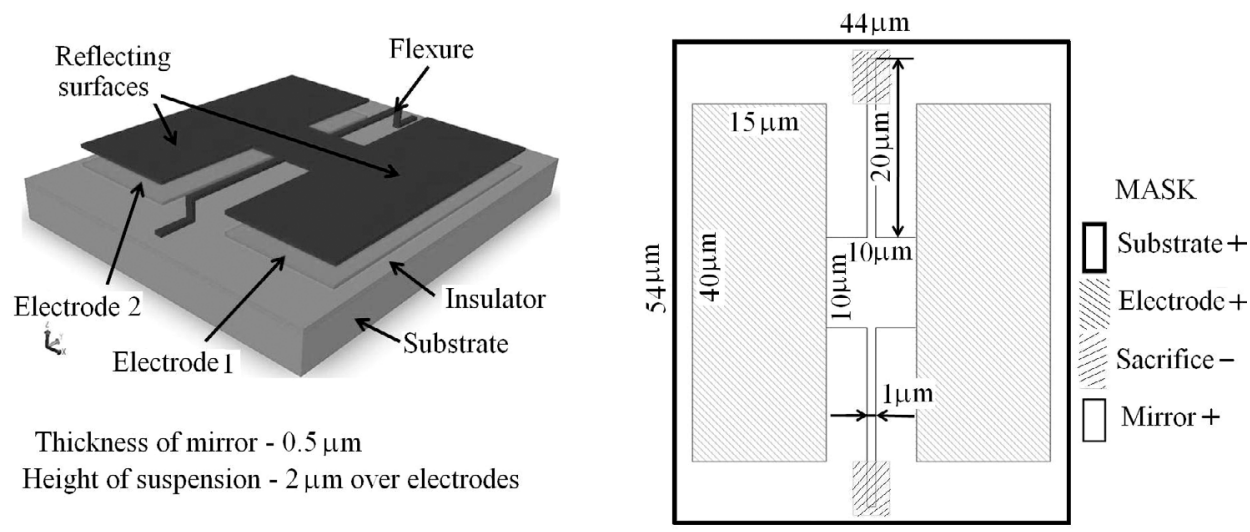

Thickness of mirror $-0.5 \mu \mathrm{m}$

Height of suspension - $2 \mu \mathrm{m}$ over electrodes

Figure 1. Geometric model of micromirror

The whole structure is built on a mono-crystalline rectangular silicon wafer of size $54 \mathrm{~mm}$ and $44 \mathrm{~mm}$. On the substrate there are deposited the following layers: insulator, electrodes and movable reflecting surfaces of the mirror. Sacrifice layer is made of polyimide and used to obtain the suspended part of micromirror. After the aluminium film is deposited the polyimide layer is removed by etching process. Constructed flexure acts as an angular joint and allows for the rotation of reflecting surfaces of micromirror. Complete sequence of subsequent manufacturing steps is presented in Table 1. For elaborated geometric model of the micromirror the FE model was created and presented in Figure 2.

Table 1. Manufacturing steps

\begin{tabular}{lllllll} 
No. & Step & Layer & Material & Thickness $[\mu \mathrm{m}]$ & Mask & Photoresist \\
\hline 0 & Substrate & Substrate & Silicon & 5 & Substrate & + \\
1 & Planar fill & Isolator & Silicon nitride & 0.5 & & \\
2 & Planar fill & Electrode & Polysilicon & 0.5 & & \\
3 & Straight cut & & & & Electrode & + \\
4 & Planar fill & Sacrifice & Polyimide & 2 & & \\
5 & Straight cut & & & Sacrifice & - \\
6 & Conformal shell & Mirror & Aluminium & 0.5 & & \\
7 & Straight cut & & & & Mirror & + \\
8 & Delete & & Polyimide & & &
\end{tabular}




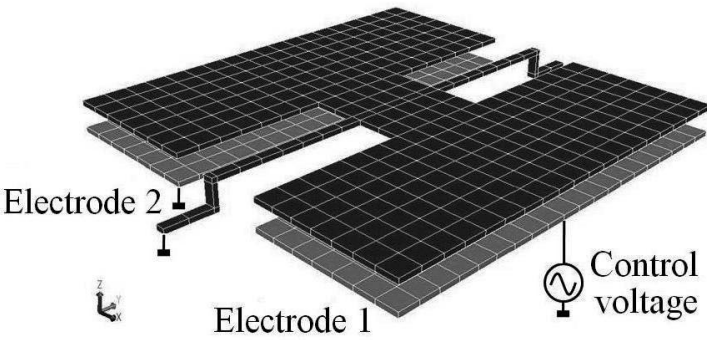

Figure 2 FE model of micromirror

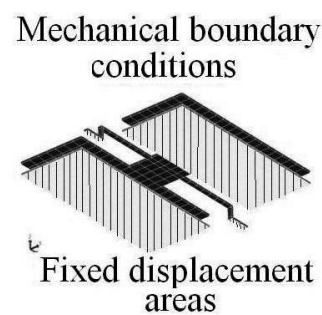

Fixed displacement areas in the FE model are bottom surfaces of electrodes and the flexure base. The angular orientation of reflecting surfaces is controlled by the voltage applied to one pair of electrodes at a time since the attraction force generated by electrostatic field depends on the difference between potentials of electrodes. In the analysis the control voltage is applied to the electrode 1 which results in the mirror rotation to the right direction. The model considers multiphysics approach and coupled analyses were performed to find its static and dynamic properties.

\section{INPUT AND OUTPUT PARAMETERS OF ANALYSIS}

As the studied output parameters, chosen static and dynamic properties of the FE model of micromirror were considered. The resultant vertical displacement DISP measured at the tips of reflecting surfaces was analyzed in case of statics (Figure 3, on the left). The nominal value of this parameter equals $D I S P_{N O M}=0.0373 \mu \mathrm{m}$. For the dynamic analysis the natural frequency $f_{1}$ related to the first normal mode of vibration was studied. Chosen normal mode is presented in Figure 3, on the right. The first normal mode was chosen since it is similar to the operational structural deflection that occurs when directing the path of light ray is performed. Knowledge on the variation of mentioned natural frequency is required to setup the parameters of controller which supplies electrodes. The nominal value of studied resonance frequency equals $f_{1, N O M}=109.7 \mathrm{kHz}$. Selected material and geometry properties as well as the control voltage were defined as the input parameters for performed calculations. Their definition and ranges of variations are described in Table 2.

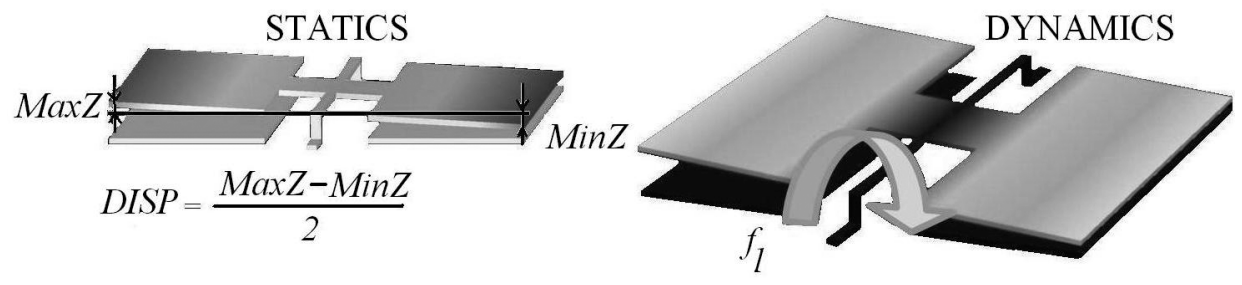

Figure 3 Output parameters of study

The whole set of input parameters was divided into two groups. The more populated one deals with uncontrollable parameters which are treated as design uncertainties. While elaboration of metamodels the relationships between both controllable and uncontrollable input and output parameters are equally important for the correct prediction of variation of chosen static and dynamic properties. In the following section there is described the application of RSM for defined input and output parameter domains. 
Table 2. Input parameters

\begin{tabular}{lllll} 
No. & Description & Symbol & Type of parameter & Range of variation \\
\hline 1 & Young's modulus of aluminium film & $E$ & Uncertain & $77 \mathrm{GPa}+/-3 \%$ \\
2 & Thickness of reflecting surfaces & $T$ & Uncertain & $0.45 \mu \mathrm{m}-0.55 \mu \mathrm{m}$ \\
3 & Voltage applied to electrode 1 & $V$ & Controllable & $0-10 \mathrm{~V}$ \\
4 & Height of suspension of reflecting surfaces over electrodes & $H$ & Uncertain & $1.9 \mu \mathrm{m}-2.1 \mu \mathrm{m}$ \\
5 & Density of aluminium film & $D$ & Uncertain & $2.3 \mathrm{~g} / \mathrm{cm}^{3}+/-3 \%$
\end{tabular}

\section{DESIGN OF EXPERIMENT, METAMODEL FITTING}

There were performed two separate procedures of metamodelling for the static and dynamic properties. For the static case the density of aluminium film was skipped. For dynamics all five input parameters were taken into account in planned Design of Experiment (DOE). DOE deals with the strategy of samples distribution within the input domain. The dimension of domain equals the number of considered input parameters. Generally the higher quality of built approximation is required the greater number of input samples should be to cover the input domain in a more dense manner. There are known both deterministic and stochastic methods of the generation of input samples. In the work the deterministic one was chosen and applied. There was assumed the use of 2-point axial design and 2nd level factorial design additionally completed with the central point. Such an approach allows for the calculation of linear, quadratic terms and introduces high order interaction terms. Detailed characteristics on applied DOE and regressors are presented in Tables 3, 4, 5 and 6, respectively to the static and dynamic case.

Table 3. DOE for static case

\begin{tabular}{llll} 
Type of DOE scheme & central point & 2-point axial design & 2nd level factorial design \\
\hline No. of required samples for $n$ parameters $(\mathrm{n}=4)$ & 1 & $2^{\mathrm{n}}$ & $2^{\mathrm{n}}$ \\
& 1 & 8 & 16 \\
Related regressors & constant term & quadratic terms & interaction terms \\
Total no. of designs & 25 & &
\end{tabular}

Table 4. Regressors for static case

\begin{tabular}{lllll} 
Type of regressor & constant & linear & 2nd to 4th order interactions & quadratic \\
\hline No. of regressors & 1 & $\mathrm{n}$ & $\left(\begin{array}{l}n \\
2\end{array}\right)+\left(\begin{array}{l}n \\
3\end{array}\right)+\left(\begin{array}{l}n \\
4\end{array}\right)$ & $\mathrm{n}$ \\
& 1 & 4 & $6+4+1=11$ & 4 \\
Total no. of regressors & 20 & &
\end{tabular}

Table 5. DOE for dynamic case

\begin{tabular}{llll} 
Type of DOE scheme & central point & 2-point axial design & 2nd level factorial design \\
\hline No. of required samples & 1 & $2 \mathrm{n}$ & $2 \mathrm{n}$ \\
for $\mathrm{n}$ parameters $(\mathrm{n}=5)$ & 1 & 10 & 32 \\
Related regressors & constant term & quadratic terms & interaction terms
\end{tabular}


Table 6. Regressors for dynamic case

\begin{tabular}{lllll} 
Type of regressor & constant & linear & 2nd to 5th order interactions & quadratic \\
\hline No. of regressors & 1 & $n$ & $\left(\begin{array}{l}n \\
2\end{array}\right)+\left(\begin{array}{l}n \\
3\end{array}\right)+\left(\begin{array}{l}n \\
4\end{array}\right)+\left(\begin{array}{l}n \\
5\end{array}\right)$ & $n$ \\
Total no. of regressors & 1 & 5 & $10+10+5+1=26$ & 5 \\
\cline { 2 - 4 } & & &
\end{tabular}

The least square method (LSM) was applied to calculate the regressors coefficients since in both cases the number of input samples is greater than the number of regressors and the problem is redundant. Obtained coefficients are presented in the form of a bar chart and shown in Figure 4 and 5, for the static and dynamic cases respectively.

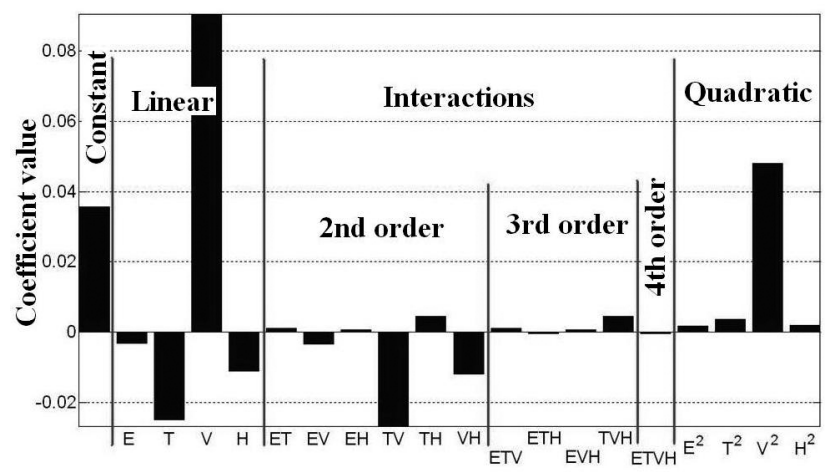

Figure 4. Coefficients of regressors calculated for the resultant static vertical displacement

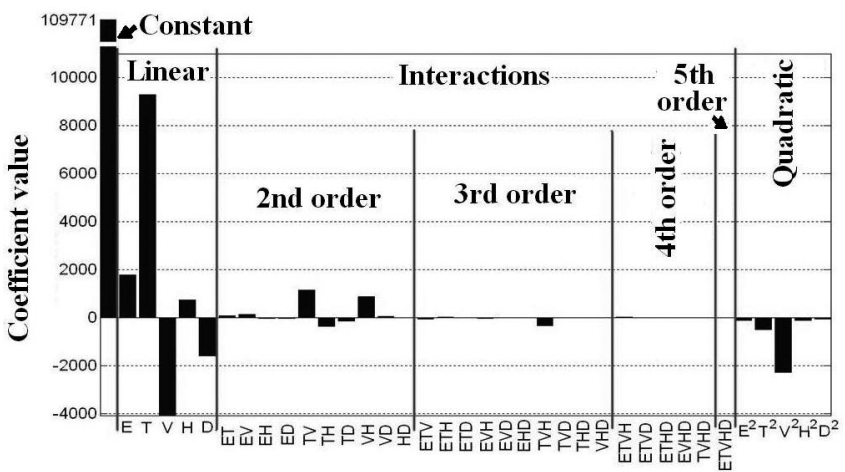

Figure 5. Coefficients of regressors calculated for the first natural frequency

Apart from the constant factor which can take any value, the following regressors seem to play important role in the metamodels: the linear and quadratic regressors related to the voltage which is especially seen for the static case as well as the thickness of reflecting surfaces and also the interactions formulated with two above mentioned parameters. For the control of mirror rotation, i.e. in the static case, there is desired possibly greatest value of 
regressor coefficients related to the voltage, preferably for those of them that have only the voltage included, i.e. $V, V^{2}, V^{3}$, etc. It leads to both high sensitivity of the rotation angle to the voltage changes and possibly the least dependency on the other parameters. For both metamodels the interactions of fourth and higher orders can be skipped. Among all regressors of the third order only one of type $T N H$ should be taken into account for both statics and dynamics. In case of material properties there are not observed any significant influences, especially for statics. Anyway the influences of both the Young's modulus and the density of aluminium film appear only for linear terms.

In the following the procedure of regressor selection to metamodels is presented. The results obtained with its application are related to the above described observations. The scheme of procedure is presented in Figure 6. The procedure is supported with genetic algorithms (GA) to be able to deal with multimodal functions. Moreover there are many successful applications of GA reported in the field of computational mechanics $[29,30,31,32]$.

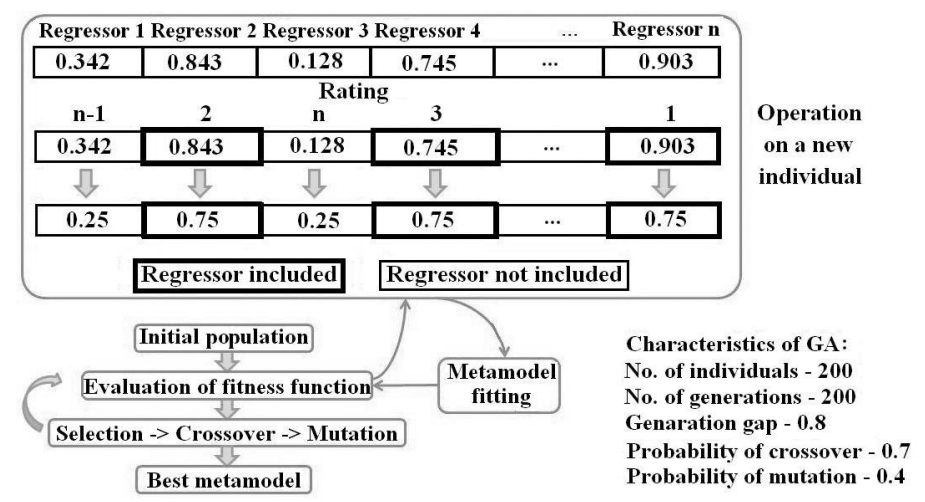

Figure 6. Procedure of regressor selection

Each individual included in a current generation consists of as many genes as the number of all considered regressors. It means the number of 20 and 37 genes for the static and dynamic case respectively as defined in Table 4 and 6. Originally, each gene can take any real value from the range $[0,1]$ but there is a procedure which forces 2 -state distinction. Genes with the highest values take a new value 0.75 . The number of these genes is assumed in the beginning and states for the number of regressors that are included in the final metamodel. The rest of genes take the value 0.25 . Hence after each sequence of operations on individuals the lower and upper threshold procedure is applied to have clear distinction between included and skipped regressors. Modified individuals are then used for the metamodel fitting procedure. Chosen regressors are only taken into account. Metamodels are created by LSM with the results of FE model simulations for assumed DOE which are defined in Table 3 and 5 respectively. For each individual there is calculated the value of fitness function, respectively $F F_{S T A T}$ or $F F_{D Y N}$, accordingly to the following formulas:

$$
\begin{gathered}
F F_{S T A T}=\max _{D O E}\left|D I S P_{A P P}-D I S P\right| \\
F F_{D Y N}=\max _{D O E}\left|f_{1, A P P}-f_{1}\right|
\end{gathered}
$$


where DISP and $f_{1}$ stand for the values of studied output parameters obtained with the results of FE analyses where as $D I S P_{A P P}$ and $f_{1, A P P}$ mean their counterparts calculated with elaborated metamodel. The same DOE are always applied. Having the value of fitness function the selection, crossover and mutation procedures are then executed. The whole sequence is repeated within the loop. The values of or $F F_{\text {STAT }}$ are $F F_{D Y N}$ are minimized until assumed number of generations is achieved. For each assumed number of included regressors the whole procedure is repeated and GA are applied separately. Figure 7 and 8 show utilized regressor selection and obtained convergence diagrams.
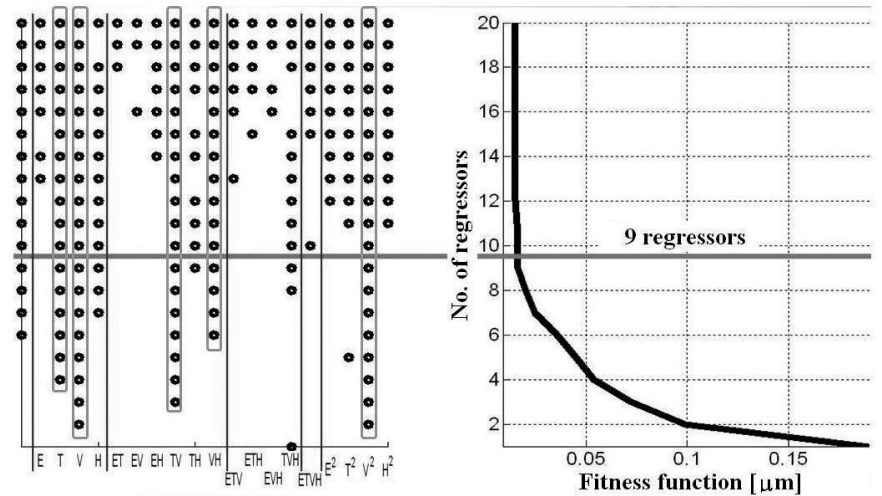

Figure 7. Regressor selection and convergence diagram for static case

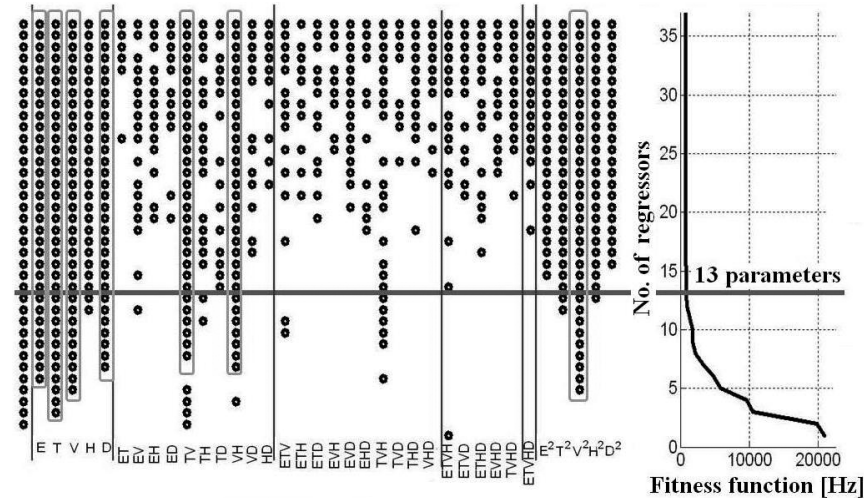

Figure 8. Regressor selection and convergence diagram for dynamic case

Bar charts presented in Figure 7 and 8 in the left show for each row separately found set of regressors which should be taken into account for assumed number of regressors. Performed optimization task minimizes the maximal difference between output parameters calculated with the FE model and the metamodel. Bar charts stand for a sort of stabilization diagrams in which it is clearly seen what are the most influential and therefore important regressors. The vertical lines made of circles, which means that given regressor is included, stabilize at first for the following regressors: $V, T, T V, V H, V^{2}$ for the static analysis and $E$, $T, V, D, T V, V H, V^{2}$ for the dynamic simulations. Above observations are coherent with the conclusions drawn from Figure 4 and 5. For both the static and dynamic output parameters 
there is observed the saturation of value of fitness function for the best fitted individuals. The saturation appears for the metamodels with 9 and 13 regressors respectively. It means that further increase of the number of included regressors results in no significant improvement of the quality of metamodel.

\section{SENSITIVITY ANALYSIS}
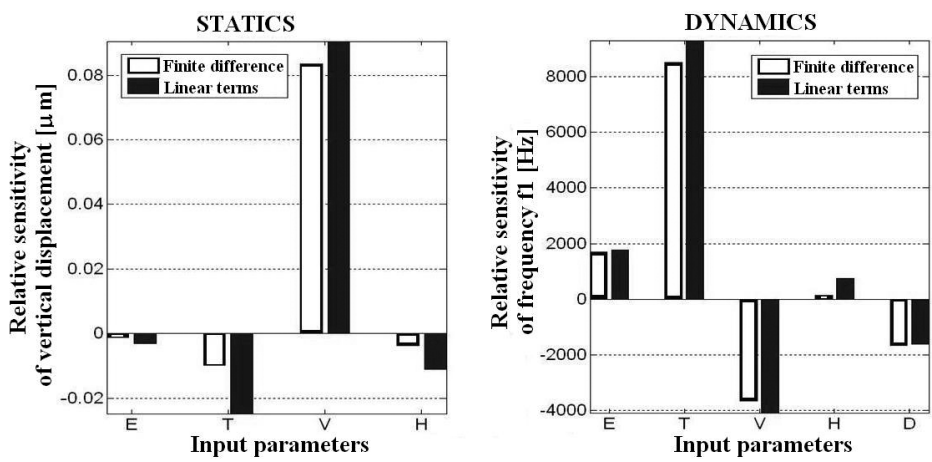

Figure 9. Sensitivities of output parameters

The sensitivity analysis was performed to verify the values of coefficients of linear regressors calculated for metamodels. Graphical comparison is present in Figure 9.

The sensitivity analysis was carried out to assess the influence of all input parameters on defined output ones. Finite difference method with central plan was applied to find the approximation of the first derivatives [19]. The results can be interpreted as the coefficients of the slope of built secants for assumed ranges of variations of input parameters. Not for all parameters and both cases the results are comparable, at least by means of the same order. The most significant differences in results appear for the suspension height $H$ and are present for both cases as well as for the thickness $T$ and seen for statics. Moreover there is a huge relative gap for the Young's modulus $E$ for the static case but eventually it seems not important because of small absolute value anyway. Presented above comparison should be treated as a qualitative one. It was made only to select the most influential parameters: the voltage $V$ which is expected especially for the static case for effective control, and the thickness of reflecting surfaces $T$. The material properties, i.e. the Young's modulus $E$ and the density $D$ are especially important for the dynamic case.

\section{UNCERTAINTY PROPAGATION}

Manufacturing of microdevices features finite quality and hence an obvious variation of material and geometry parameters appearing for subsequent items of produced components. A design process for MEMS devices should then take into account present uncertainties to enable more realistic results of numerical simulations. Therefore in present work the preliminary uncertainty analysis with a limited number of samples was carried out for modelled micromirror. The propagation of variation of all input parameters for the output parameters was analysed. For this analysis all parameters were allowed to vary within their assumed ranges independently to whether they were treated as uncertain or control ones. The overall variation of output parameters was of concern. Deterministic DOE with uniformly distributed samples was assumed to cover the input domain. In case of the static analysis the 
total number of samples equals 297. Three values, i.e. the nominal, the lower and upper bounds accordingly to data presented in Table 2, were considered for the Young's modulus $E$, the thickness $T$ and the height of suspension $H$. More dense distribution was assumed for the control voltage $V$, allowing for the change of $1 \mathrm{~V}$ in the range $0-10 \mathrm{~V}$. In case of the dynamic analysis there were assumed only three values (the nominal, the lower and upper bounds) independently taken by each of 5 input parameters. It results in 243 samples to be checked. There were compared the results obtained with:

- $\quad$ FE analyses

- metamodels with all regressors (20 for statics and 37 for dynamics)

- metamodels with reduced number of regressors (9 for statics and 13 for dynamics as shown in Figure 7 and 8)

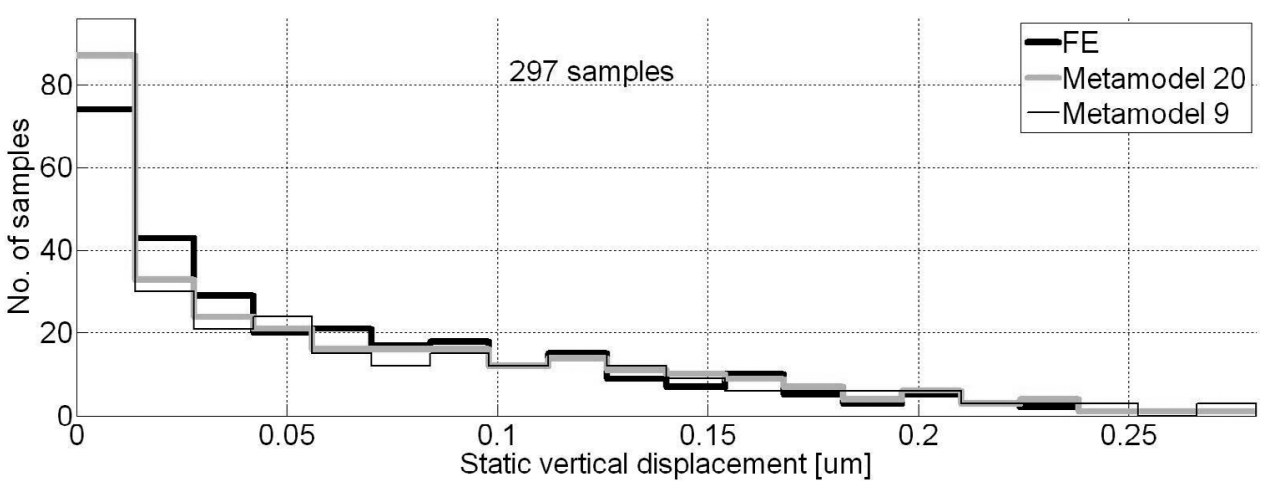

Figure 10. Histogram of the resultant static vertical displacement

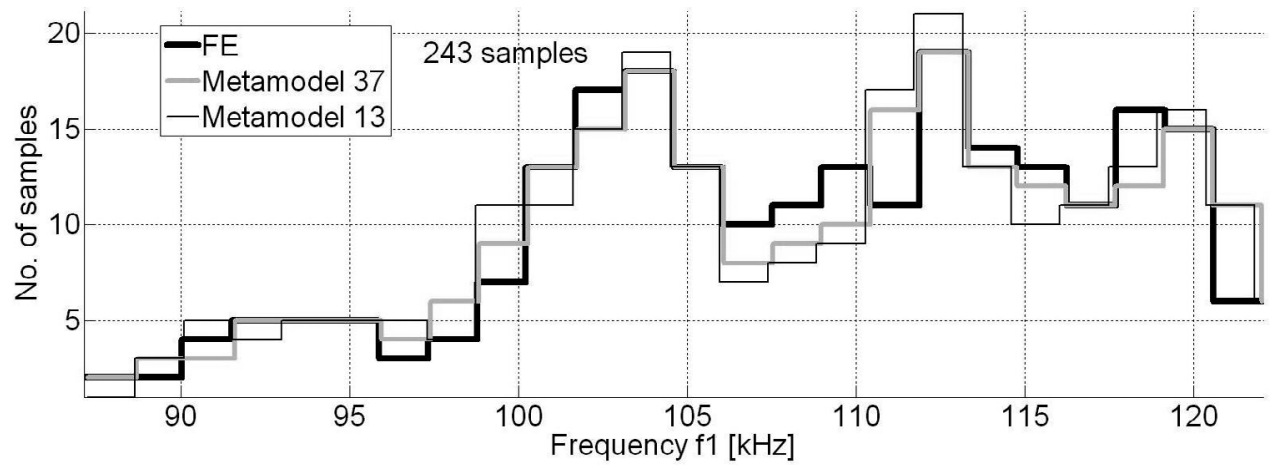

Figure 11. Histogram of the first natural frequency

Obtained histograms are presented in Figure 10 and 11. The numerical characteristics of variation of output parameters is presented in Table 7.

In case of the metamodels elaborated for statics the artefact appears that negative values of resultant vertical displacement are present for selected samples. Their values are however 2 orders smaller than the maximal displacement and obviously should be treated as resulting from metamodel inaccuracy. In case when no voltage is applied to the electrodes there is no 
flexure deflection and the angle of rotation equals zero. For assumed DOE the mean value and the variance of resultant displacement calculated with metamodels are slightly overestimated. For the first natural frequency there are observed small overestimation concerning the variance. For both the static and dynamic case it is also observed that the convergence of minimal and maximal values to the respective results obtained in the FE analyses is better in case when greater number of regressors is introduced.

Table 7. Variation of output parameters

\begin{tabular}{lccccc} 
Case/Parameter & Model & Mean & Variance & Min & Max \\
\hline Statics/ Resultant & FE & $0.0583 \mu \mathrm{m}$ & $0.0037 \mu \mathrm{m}^{2}$ & $0 \mu \mathrm{m}$ & $0.2802 \mu \mathrm{m}$ \\
\cline { 2 - 6 } vertical displacement & Metamodel 20 & $0.0600 \mu \mathrm{m}$ & $0.0043 \mu \mathrm{m}^{2}$ & $-0.0068 \mu \mathrm{m}$ & $0.2762 \mu \mathrm{m}$ \\
\cline { 2 - 6 } & Metamodel 9 & $0.0589 \mu \mathrm{m}$ & $0.0044 \mu \mathrm{m}^{2}$ & $-0.0083 \mu \mathrm{m}$ & $0.2644 \mu \mathrm{m}$ \\
\hline Dynamics/ First & FE & $107.8 \mathrm{kHz}$ & $71.8 \mathrm{kHz}^{2}$ & $86.4 \mathrm{kHz}$ & $122.8 \mathrm{kHz}$ \\
\cline { 2 - 6 } natural frequency & Metamodel 37 & $107.7 \mathrm{kHz}$ & $75.5 \mathrm{kHz}^{2}$ & $86.5 \mathrm{kHz}$ & $122.7 \mathrm{kHz}$ \\
\cline { 2 - 6 } & Metamodel 13 & $107.7 \mathrm{kHz}$ & $75.5 \mathrm{kHz}^{2}$ & $86.5 \mathrm{kHz}$ & $122.5 \mathrm{kHz}$ \\
\hline
\end{tabular}

\section{SUMMARY AND CONCLUDING REMARKS}

In the paper there is presented the application of RSM to static and dynamic analyses carried out for the FE model of micromirror. Elaborated metamodels were used for the analysis of variation of selected static and dynamic properties of microdevice. Approximating models were applied to study the sensitivity of output parameters and to assess the overall propagation of variation assumed for the uncertain and control parameters. The multiphysics approach was used to consider different physical phenomena. Apart from a mechanical part related to structural dynamics, the electrostatic field was introduced to control the rotation of reflecting surfaces of micromirror. As the input parameters there were defined 5 quantities related to geometry, material properties and control activity.

Parameterized FE model of micromirror was used to study the quality of metamodels depending on the number of introduced regressors. The procedure of regressor selection was based on GA and allowed to find metamodel that characterizes the best agreement with the results of FE analyses. For both the static and dynamic output parameters the saturation of values of fitness function for the best fitted individuals was observed. It means that further increase of the number of included regressors resulted in no significant improvement of the quality of metamodel. For applied metamodels the preliminary uncertainty analysis with limited number of samples was carried out to compare the results with the outcomes from the FE simulations. Slight overestimation of the results was noticed. However created metamodels allowed to built the histograms describing the variation of output parameters correctly.

The sensitivity analysis was carried out to assess the influence of all input parameters on defined output ones. The contribution of different regressors in approximating functions for the static displacement and chosen natural frequency was also analysed. The thickness and the voltage were found as the most influential input parameters. Significant influence of the quadratic term for voltage was observed which should be taken into account while elaboration the control system.

\section{REFERENCES}

1. Allen J.J., Micro electro mechanical system design, Mechanical engineering 192, CRC Press, 2005.

2. Bao M., Analysis and design principles of MEMS devices, Elsevier Science, 2005. 
3. Lin R.M., Wang W.J., Structural dynamics of microsystems - current state of research and future directions, Mechanical Systems and Signal Processing, 2006, 20, 1015-1043.

4. Gianchandani Y., Tabata O., Zappe H., Comprehensive Microsystems, Elsevier Science, 2007.

5. Mawardi A., Pitchumani R., Design of microresonators under uncertainty, Journal of Microelectromechanical Systems, 2005, 14, 63-69.

6. Batra R.C., Porfiri M., Spinello D., Review of modeling electrostatically actuated microelectromechanical systems, Smart Materials and Structures, 2007, 16, R23-R31.

7. Liu M., Maute K., Frangopol D.M., Multi-objective design optimization of electrostatically actuated microbeam resonators with and without parameter uncertainty, Reliability Engineering and System Safety, 2007, 92, 1333-1343.

8. Martowicz A., Uhl T., Klepka A., Application of multiphysics approach to study variation of dynamic properties of MEMS structure, Proc. of ISMA 2010 Conference on Advanced Acoustics and Vibration Engineering, Leuven, Belgium, September 20-22, 2010.

9. Ergun A.S., Huang Y., Zhuang X., Oralkan O., Yaralıglu G.G., Khuri-Yakub B.T., Capacitive micromachined ultrasonic transducers: fabrication technology, IEEE Transactions on Ultrasonics, Ferroelectrics, and Frequency Control, 2005, 52(12), 2242-2258.

10. Judy J.K., Microelectromechanical systems (MEMS): fabrication, design and applications, Smart Materials and Structures, 2001, 10(6), 1115-1134.

11. Wong S.H., Kupnik M., Zhuang X., Lin D.-S., Butts-Pauly K., Khuri-Yakub B., Evaluation of wafer bonded CMUTs with rectangular membranes featuring high fill factor, IEEE Transactions on Ultrasonics, Ferroelectrics, and Frequency Control, 2008, 55(9), 2053-2065.

12. Martowicz A., Stanciu I., Uhl T., Uncertainty analysis for dynamic properties of MEMS resonator supported by fuzzy arithmetics, The International Journal of Multiphysics, 2009, 3(3), 201-219.

13. Uhl T., Martowicz A., Codreanu I., Klepka A., Analysis of uncertainties in MEMS and their influence on dynamic properties, Archives of Mechanics, 2009, 61(5), 349-370.

14. Codreanu I., Martowicz A., Gallina A., Pieczonka L., Uhl T., Study of the effect of process induced uncertainties on the performance of a micro-comb resonator, Solid State Phenomena, 2009, 147-149, 716725 .

15. Mir S., Charlot B., Courtois B., Extending fault-based testing to micromechanical systems, Journal of Electronic Testing: Theory and Applications, 2000, 16, 279-288.

16. Montgomery D.C., Runger G., Applied statistics and probability for engineers, JohnWiley and Sons, New York, 2007.

17. Myers R.H., Montgomery D.C., Response Surface Methodology process and product optimization using designed experiments, John Wiley \& Sons, Inc., New York, 1995.

18. Helton J.C., Davis F.J., Latin hypercube sampling and the propagation of uncertainty in analyses of complex systems, Reliability Engineering and System Safety, 2003, 81(1), 23-69.

19. Kleiber M., Antunez H., Hien T.D., Kowalczyk P., Parameter sensitivity in nonlinear mechanics, John Wiley \& Sons, Chichester, England, 1997.

20. Schueller G.I., Uncertainty \& reliability analysis of structural dynamical systems, Mota Soares C.A., Martins J.A.C., Rodrigues H.C., Ambrósio J.A.C. [Eds.], Computational Mechanics. Solids, Structures and Coupled Problems, Springer, Dordrecht, The Netherlands, 541-554, 2006.

21. Tiso P., Rixen D.J., Reduction methods for MEMS nonlinear dynamics analysis, Proc. of the IMAC-XXVIII, Jacksonville, USA, February 1-4, 2010.

22. Martowicz A., Uhl T., Assessment of variation of natural frequencies of FE model based on the application of alpha-cut strategy and genetic algorithms, Finite Element in Analysis and Design, 2011, 47(1), 63-71.

23. Johnson E.A., Proppe C., Spencer B.F. Jr., Bergman L.A., Szekely, G.S. Schueller G.I., Parallel processing in computational stochastic dynamics, Probabilistic Engineering Mechanics, 2003, 18, 37-60.

24. Burczynski T., Orantek P., The two-stage fuzzy strategy in identification of the uncertain boundary conditions, Proc. of AI-METH 2005 - Artificial Intelligence Methods, Gliwice, 43-51, November 16-18, 2005 . 
25. Kudrle T.D., Wang C.C., Bancu M.G., Hsiao J.C., Pareek A., Waelti M., Kirkos G.A., Shone T., Fung C.D., Mastrangelo C.H., Single-crystal silicon micromirror array with polysilicon flexures, Sensors and Actuators A, 2005, 119, 559-566.

26. Min Y.-H., Kim Y.-K., Modeling, design, fabrication and measurement of a single layer polysilicon micromirror with initial curvature compensation, Sensors and Actuators, 1999, 78, 8-17.

27. Zhou G., Cheo K.K.L., Tay F.E.H., Chau F.S., Neural network approach for linearization of the electrostatically actuated double-gimballed micromirror, Analog Integrated Circuits and Signal Processing, 2004, 40, 141-153.

28. http://www.coventor.com/

29. Deb K., Gulati S., Design of truss-structures for minimum weight using genetic algorithms, Finite Elements in Analysis and Design, 2001, 37(5), 447-465.

30. Dias J.P., Correa R.M., Multiobjective optimization of multibody systems with genetic algorithms, Proc. of III European Conference on Computational Mechanics, Lisbon, June 5-8, 2006.

31. Martowicz A., Pieczara J., Uhl T., Application of soft computing in uncertainty analysis carried out within structural dynamics, Computer Assisted Mechanics and Engineering Sciences - CAMES, 2007, 14, 293-305.

32. Martowicz A., Pieczonka L., Uhl T., Application of uncertainty analysis in structural dynamics, Journal of KONES Powertrain and Transport, 2008, 15(1), 153-167. 
